\title{
IL-1 Induces Fascin Expression and Increases Cancer Invasion
}

\author{
MIN KYEONG LEE, JI HYEON PARK, SEOL HWA GI and YOUNG SUN HWANG \\ Department of Dental Hygiene, College of Health Science, \\ Eulji University, Seongnam, Republic of Korea
}

\begin{abstract}
Background/Aim: Tumor microenvironment plays an important role in tumor growth and metastasis. Cancer cells can promote their growth and malignancy by altering the surrounding stroma. Fascin is an actin-bundling protein that regulates the dynamics of the cytoskeletal structure and plays a significant role in cancer invasion and metastasis. In this study, we observed stromal factors controlling fascin expression in cancer cells and investigated underlying signal transduction pathways. Materials and Methods: Fascin depletion was performed with lentiviral short-hairpin RNA (shRNA) against fascin $m R N A$ and a stable cell line $\left(\right.$ Fascin $\left.^{\text {dep }}\right)$ was established. Fascin expression and invasion activity induced by $I L-1 \beta$ treatement were observed through Matrigel-Transwell invasion and $3 D$ culture system. Intermediated signaling molecules involved in fascin expression induced by $I L-1 \beta$ were elucidated using western blotting. Results: Fascin was more highly expressed in human OSCC cells than normal cells. Cancer invasion activity was decreased by fascin depletion using lentiviral shRNA. However, fascin expression was increased by $I L-1 \beta$ treatement, leading to increased extracellular matrix (ECM) degradation and infiltration into 3-dimensional (3-D) collagen matrix. Specific inhibitors of extracellular signalregulated kinases-1/2 [ERK1/2, (PD98059)], c-Jun Nterminal kinase [JNK, (SP600125)], nuclear factor kappa light chain enhancer of activted $B$ cells [NF-KB, (parthenolide)], and cAMP response element binding protein [CREB, (CREB inhibitor)] suppressed IL-1 $\beta$-induced fascin expression. IL-1 $\beta$ induced phosphorylation of ERK1/2, JNK, $N F-K B$ and CREB while IL-1 receptor (IL-1R) antagonist abolished their activation. Conclusion: $I L-1 \beta$ is a critical inducer of fascin expression. ERK1/2,JNK, NF-KB, and CREB signaling pathways are involved in $I L-1 \beta$-induced
\end{abstract}

Correspondence to: Y.S. Hwang, Department of Dental Hygiene, College of Health Science, Eulji University, 553 Sansung-Daero, Soojung-Gu, Seongnam, Gyunggi-Do, 13135, Republic of Korea. Tel: +82 37407493, Fax: +82 37407352, e-mail: kiteys@eulji.ac.kr

Key Words: IL-1 $\beta$, fascin, cancer invasion, 3D culture, antagonist. fascin expression and these paracrine signaling pathways can induce cancer cell invasion.

Cancer growth and invasiveness are closely related to cancer progression and prognosis. Invasive cancer cells from the primary site can be released from the adjucent tissues by secreation of proteolytic enzymes capable of degrading the surrounding exracellular matrix (ECM) and spread to secondary sites. Therefore, focalized protease release to nearby stroma in the direction of cancer invasion is crucial to enhance invasion efficiency (1). Cancer cells can form a protrusive cellular structure called invadopodia for invasion. A complete invadopodia assembly is a very important process for cancer invasion activity $(2,3)$. When infiltration is needed, cancer cells induce actin polymerization based on Arp2/3 complex and N-WASP to stabilize the protruding structure by joining many molecules such as Tsk5, cortactin, synaptjanin-2, Abl-family kinase Arg4, and integrin. When the protease is secreted intensively around the protruding structure, the extracellular matrix is deeply degraded, acting as a mechanism to increase cancer cell invasion $(2,4)$. Recent studies have indicated that invadopodia is regulated by tumor environment signals including growth factors (2, 5), hypoxia (6), pH (7), metabolism (8), and stromal cell interactions (9).

Initiation of invadopodia formation can be induced by growth factors such as epidermal growth factor (EGF), transforming growth factor beta (TGF- $\beta$ ), and plateletderived growth factor (PDGF) (10). These results suggest that invadopodia formation can be regulated by paracrine signaling pathways in cancer microenvironment. Thus, the influence of tumor surrounding microenvironment on cancer invasion should also be studied. Fascin is an actin-bundling protein highly expressed in various cancer tissues. Fascin is localized in the invadopodia and is known to facilitate ECM invasion (11). Despite the fact that invadopodia formation can be induced by various factors, there is little research on the regulation of fascin expression. Therefore, the objective of this study was to determine the effect of IL- $1 \beta$ stimulation on fascin expression and invasion activity and elucidate related cell signaling processes. 


\section{Materials and Methods}

Cell culture. HSC-3 OSCC cells was grown in DMEM/F12 (3:1 ratio) medium supplemented with $10 \% \mathrm{FBS}, 1 \times 10^{-10} \mathrm{M}$ cholera toxin, $0.4 \mathrm{mg} / \mathrm{ml}$ hydrocortisone, $5 \mu \mathrm{g} / \mathrm{ml}$ insulin, $5 \mu \mathrm{g} / \mathrm{ml}$ apotransferrin, and $2 \times 10^{-11} \mathrm{M}$ triiodothronine (T3) in a humidified atmosphere of $5 \% \mathrm{CO}_{2}$ at $37^{\circ} \mathrm{C}$. The high invasive variant HSC-3 cell subline was obtained by serial selection through Matrigelcoated Transwell invasion.

Reagents. All reagents used in cell culture were purchased from Gibco BRL Co. (Rockville, MD, USA). Cholera toxin, hydrocortisone, insulin, apo-transferrin, T3, dimethyl sulfoxide (DMSO), and parthenolide were obtained from Sigma Chemical Co. (St. Louis, MO, USA). Recombinant human interleukin 1 beta (IL$1 \beta$ ) was purchased from R\&D Systems (Minneapolis, MN, USA). PD98059, SP600125, and CREB inhibitor (666-15) were purchased from Calbiochem (La Jolla, CA, USA). Interleukin 1 receptor (IL1R) antagonist was purchased from Cayman (Cayman Chemical, Ann Arbor, MI, USA). The following antibodies were purchased from their respective sources: Fascin (Dako, Carpentaria, CA, USA); total/phospho form of ERK, JNK, NF-KB, and CREB (Cell Signaling Technology, Danvers, MA, USA); actin (Sigma). Oregon Green 488 gelatin were purchased from Molecular Probes (Carlsbad, CA, USA).

Fascin depletion. Fascin 1-specific shRNA (h) lentiviral particles were transduced in cultured cell with $5 \mu \mathrm{g} / \mathrm{ml}$ polybrene according to the manufacturer protocol (Santa Cruz, CA, USA). Continual selection was followed with $1 \mathrm{mg} / \mathrm{ml}$ puromycin to establish fascindepleted stable cell line. Control shRNA (h) lentiviral particles-A (Santa Cruz) was also used as a negative control. The extent of fascin depletion was evaluated by western blot.

Western blotting. Proteins $(50 \mu \mathrm{g})$ were separated on SDSpolyacrylamide gel and were transferred to a polyvinylidene difluoride membrane (Millipore, Billerica, MA, USA). The membrane was blocked with $10 \%$ skim milk in PBS containing $0.1 \%$ Tween-20 (PBS-T) and subsequently incubated overnight with a 1:1,000 dilution of primary antibody against its specific protein at $4^{\circ} \mathrm{C}$. The blots were then incubated with a 1:3,000 dilution of their respective horseradish peroxidase-conjugated secondary antibodies for $2 \mathrm{~h}$ at room temperature and were washed with PBS-T. The targeted proteins were visualized using an enhanced chemiluminescence detection kit (Amersham Life Science, Arlington Heights, IL, USA) according to the manufacturer's instructions.

RT-PCR. Total RNA was isolated using TRIzol reagent (Life Technologies, Foster City, CA, USA) according to the instructions provided by the manufacturer. Reverse transcription to obtained cDNA and amplification by PCR using specific primer pairs. Fascin 1 (GenBank accession No. NM_001100806) primer; sense 5'GTCCACTGCATCCACTAAGA-3' and anti-sense 5'-AGCTGA AAGACGTCGTAACT-3' (Tm $55^{\circ} \mathrm{C}, 276$ base pairs), GAPDH primer; sense 5'- GAAGGTGAAGGTCGGAGTC-3' and anti-sense 5'- GAAGATGGTGATGGGATTTC-3' (Tm $57^{\circ} \mathrm{C}, 250$ base pairs). The reaction products were electrophoresed on $1 \%$ agarose gel.

Invasion assay. 8- $\mu \mathrm{m}$ pore sized polycarbonate nucleopore filter inserts in a 24-well Transwell chamber (Corning Costar, Cambridge, MA, USA), were coated with Matrigel (30 $\mu \mathrm{g} / \mathrm{well}$; Becton
Dickinson, Lincoln Park, NJ, USA). Cells ( $5 \times 10^{4}$ cells) were added into the upper chamber, and complete medium was added to the bottom chamber and kept for $48 \mathrm{~h}$ at $37^{\circ} \mathrm{C}$. Invaded cells on the lower surface of the membrane were fixed with ethanol and noninvasive cells were removed with a cotton swab. Then cells were stained with hematoxylin. Invaded cells from five fields were counted under a microscope.

ECM degradation. Oregon Green 488 gelatin-coated coverslips were prepared as described previously (12). Cells $\left(3 \times 10^{3}\right.$ cells $)$ were plated on coverslips in 12-well plates and cultured for $16 \mathrm{~h}$. Cells were fixed with $4 \%$ paraformaldehyde followed by permeabilization with $0.5 \%$ Triton X-100/PBS and stained for nuclei with DAPI. Areas of matrix degradation was identified by a loss fluorescence using an EVOS FL monochrome microscope (ThermoFisher Scientific, Waltham, MA, USA).

Three-dimensional culture. A dermal equivalent gel was generated with a Type I-A collagen mixture with eight volumes of ice-cold collagen solution (Nitta Gelatin Inc, Osaka, Japan), one volume of $10 \times$ reconstitution solution $(0.022 \mathrm{~g} / \mathrm{ml} \mathrm{NaHCO} 3,0.0477 \mathrm{~g} / \mathrm{ml}$ HEPES, $0.05 \mathrm{~N} \mathrm{NaOH}$ ), and one volume 10× DMEM. Immortalized gingiva fibroblast $\left(1 \times 10^{5}\right.$ cells) was added to collagen mixture (13, 14). This mixture was poured onto polycarbonate filter inserts (3$\mu \mathrm{m}$ pore size, $12 \mathrm{~mm}$ diameter; Millipore) and placed in 6-well plates. After $24 \mathrm{~h}$ incubation at $37^{\circ} \mathrm{C}$, HSC- 3 cell $\left(1 \times 10^{5}\right.$ cells $)$ was loaded on collagen gel and medium was added to the 6 -well plates. The culture medium was then changed every 2-3 days for 2 weeks. Gels were then formalin-fixed, paraffin-embedded and histologically examined. To measure invasive areas and depth, the culture tissue was stained with hematoxylin and eosin (H\&E).

Statistical analysis. The statistical analysis was performed using InStat GraphPad Prism ver. 5.01 statistical software (GraphPad Software, Inc., San Diego, CA, USA). Results are expressed as mean \pm standard deviation. $p$-Values $<0.05$ were considered significant.

\section{Results}

Fascin is involved in cancer cell invasion. Surgical tissues from patients with oral squamous cell carcinoma (OSCC) and adjacent normal epithelia were used to analyze fascin expression. As shown in Figure 1A, fascin expression level was higher in cancer than that in normal tissue. As a result of the experiment utilising biological-specific inhibitors, fascin mRNA expression was inhibited by PD98059, SP600125, parthenolide, and CREB inhibitor (Figure 1B). This result indicates that extracellular signal-regulated kinases (ERK1/2) and c-Jun N-terminal kinase (JNK) act as intermediate signaling molecules and NF-KB (nuclear factor kappa light chain enhancer of activted B cells) and CREB (cAMP response element binding protein) are associated with fascin expression (Figure 1B). To confirm the role of fascin in cancer invasion, fascin down-regulated cell line $\left(\right.$ Fascin $^{\text {dep }}$ ) was established using lentiviral shRNA (Figure 1C). In Matrigel-coated Transwell invasion, Fascin ${ }^{\text {dep }}$ cells showed 7.2-times lower invasion activity compared to control (Figure 1D). 

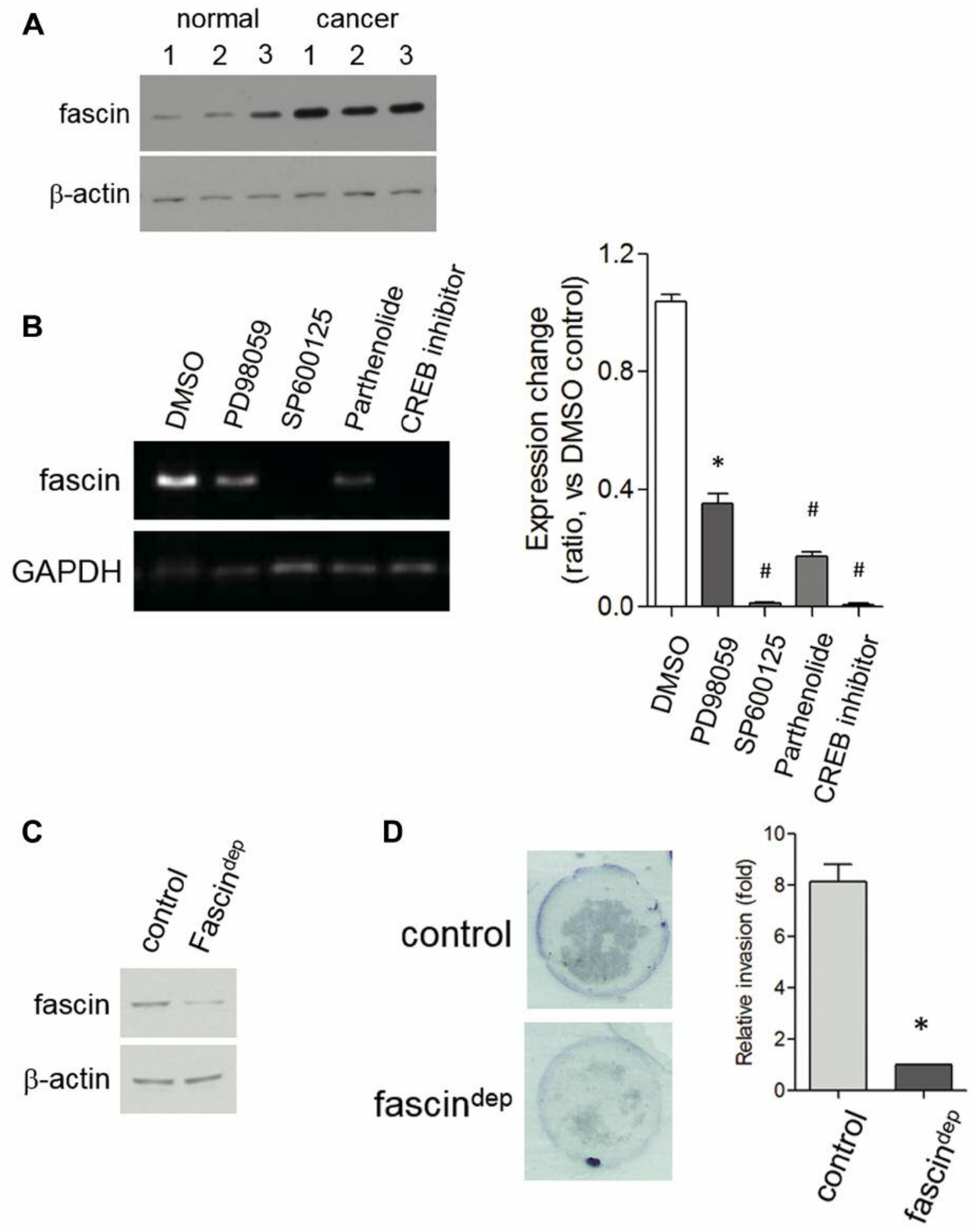

Figure 1. Fascin expression and invasion. A: Fascin Expression in normal and cancer cells was analysed by western blot. $\beta$-actin was detected as a loading control. B: Fascin mRNA expression in HSC-3 OSCC cells with or without biological-specific inhibitors was analyzed by RT-PCR. GAPDH level was used for $m R N A$ loading control. Relative changes of $m R N A$ expression were plotted. ${ }^{*} p<0.01$, \#p<0.001 versus DMSO control. C: Fascindepleted cell (Fascindep) was prepared using fascin shRNA lentiviral particles and puromycine resistance clone was analyzed by western blot for reduction of fascin level. Control shRNA lentiviral particles-A was used to prepare a control stable cell line (control). $\beta$-actin was used as loading control. D: Matrigel-coated Transwell invasion activity of Fascindep cells. Invasion assay was proceeded for 48 h. Invading cells were detected by hematoxylin staining and counted. $* p<0.001$ versus control cells. 


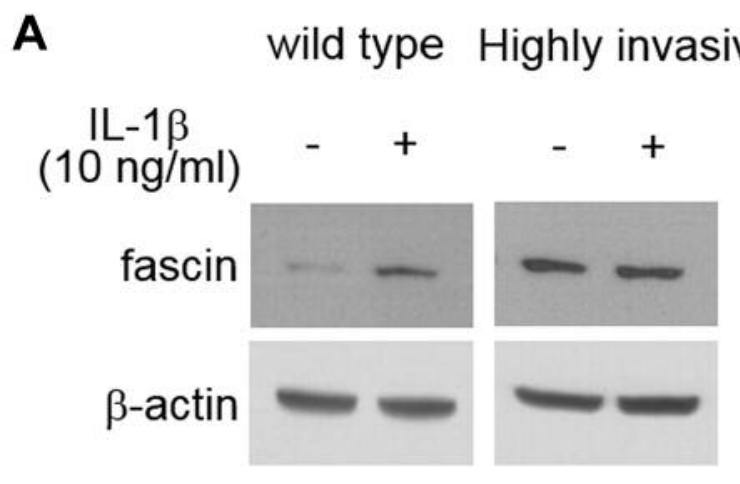

B

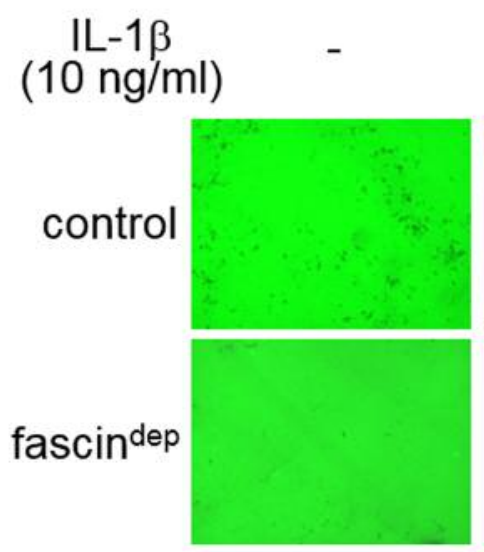

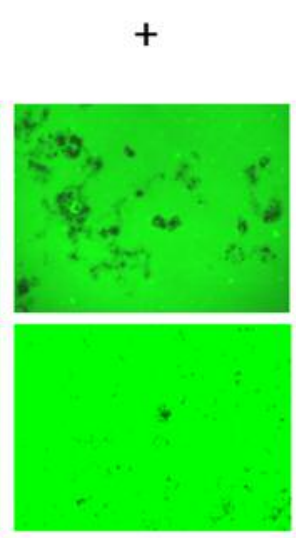

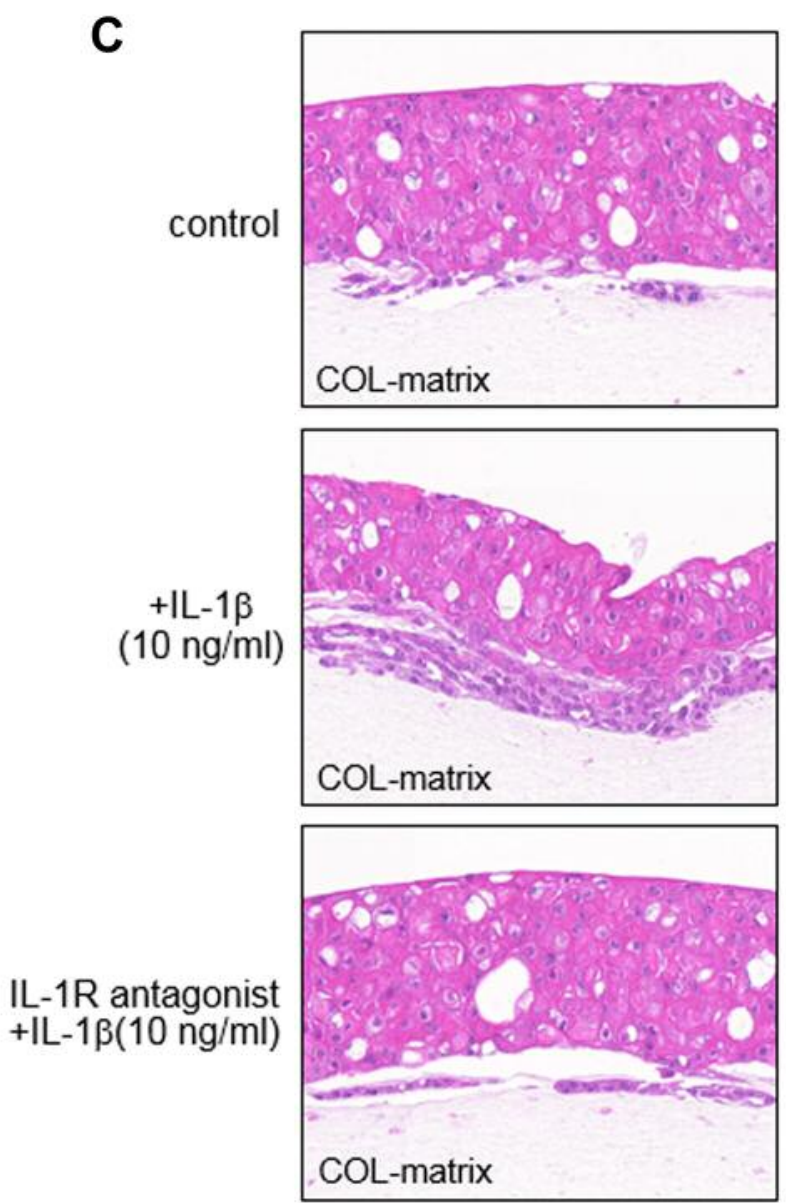

Figure 2. Effect of IL-1 $\beta$ on fascin expression and cell invasion. A: Fascin expression by IL-1 $\beta$ was analyzed in wild-type and highly invasive cells. $\beta$-actin was detected as a loading control. B: Matrix degradation activity of Fascindep cells. Cells were cultured on fluorophore (FITC)-conjugated gelatin matrix for $12 \mathrm{~h}$. Degraded matrix is shown as dark areas. C: IL-1 $\beta$ effect on $3 D$ invasion. Wild type cells on Type I-A collagen mixture (COL-matrix) were culture for 10 days. Invasion of cells within the dermal equivalent was analyzed by H\&E staining.

$I L-1 \beta$ increases fascin expression and cancer invasion. To investigate changes in fascin expression induced by IL-1 $\beta$ stimulation, cells were treated with $10 \mathrm{ng} / \mathrm{ml} \mathrm{IL}-1 \beta$ for $8 \mathrm{~h}$, followed by western blot analysis. As shown in Figure 2A, fascin expression was increased by IL- $1 \beta$ stimulation in the wild-type control. This increase was not induced in highly invasive cells isolated via serial selection through Transwell invasion. It is presumed that fascin expression is sufficiently high in highly invasive cells. In addition, IL- $1 \beta$ stimulation increased the degradation activity of FITC-gelatin matrix in control cells (Figure 2B). However, the extent of IL-1 $\beta$ induced matrix degradation in $\operatorname{fascin}^{\Delta}$ cell was not as large as in the wild type control. Consistent with this result, invasive areas into dermal equivalents (COL-matrix) were deeply extended by IL-1 $\beta$ stimulation in three-dimensional (3-D) culture (Figure 2C).
ERK1/2, JNK, NF-KB, and CREB signaling molecules are involved in $I L-1$-induced fascin expression. IL- $1 \beta$ receptor (IL-1R) antagonist treatment inhibited such invasive area expansion induced by IL-1 stimulation. IL- $1 \beta$-induced phosphorylation of ERK $1 / 2$ and JNK were reduced by IL-1R antagonist (Figure 3). IL-1 $\beta$-induced phosphorylation of NF$\mathrm{KB}$ and CREB was also reduced by IL-1R antagonist. These results indicate that ERK1/2 and JNK can act as intermediate signaling molecules in IL-1 $\beta$-induced fascin expression and NF-KB and CREB serve as transcripton factors.

\section{Discussion}

Stroma plays a structural and connective role in tissues. It consists of basement membrane, fibroblasts, extracellular matrix, immune cells and vascular system. Normal stroma 


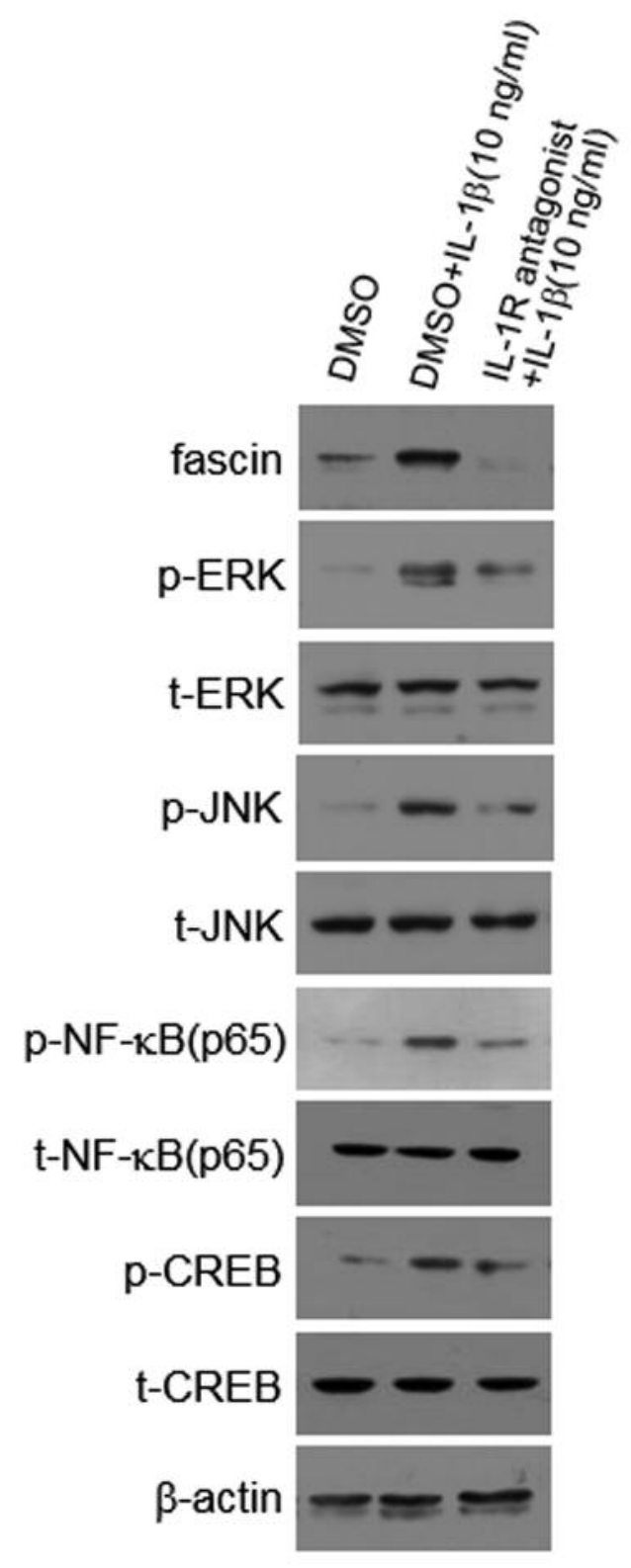

Figure 3. Analysis of intermediate signaling molecules for $I L-1 \beta$-induced fascin expression. Wild-type cells were treated with $I L-1 \beta$ for $30 \mathrm{~min}$ (analysis for total/phospho-ERK and total/phospho-JNK) or $1 \mathrm{~h}$ (analysis for total/phospho-NF-KB and total/phospho-CREB) and western blot were performed with specific antibody. IL-1R antagonist was pretreated for 1 $h$ before IL-1 $\beta$ treatment. $\beta$-actin was detected as loading control.

maintains homeostasis by inhibiting inflammation and neoplasia through immune systems. However, in the tumor environment, it plays a role in promoting cancer growth and malignant tumors (15). Cancer cells produce several factors such as transforming growth factor- $\beta$ that can cause differentiation of normal fibroblasts into carcinoma-associated fibroblasts (CAF), thereby serving as a pivotal inducer of tumor growth, invasion and metastasis $(16,17)$. CAF also secrete various chemokines and cytokines that can affect tumor and other stromal cells, leading to cancer growth and invasion. Therefore, the reciprocal cross-talk between stromal tissue and tumor plays a pivotal role in cancer growth and progression.

IL-1 $\beta$ is a member of the interleukin (IL) 1 family of cytokines. This cytokine is an important mediator of the inflammatory response. It is involved in a variety of cellular activities including cell proliferation, differentiation, and apoptosis (18). Many studies have shown that IL-1 is highly expressed in many tumor types. It is involved tumor progression by modulating several factors related to metastatic and angiogenic genes. Patients with IL-1-producing tumors generally have poor prognoses (19). In addition, high IL-1 level within the tumor stroma is associated with a virulent tumor phenotype (20). Therefore, anti-IL-1 agents are proposed as therapeutic agents. Despite progress from many studies, the exact mechanism of IL-1 involvement in tumor growth and progression remains unclear. Here, we observed cellular mechanisms of IL-1-induced cancer invasion. As a result, IL-1 increased fascin expression. Suppression of fascin expression significantly reduced cancer cell invasion. Fascin localizes in invadopodia and stabilizes actin polymerization (11). These data suggest that IL-1 can promote stabilization of invadopodia by increasing fascin expression, thereby aggravating cancer invasion. An increase in cancer invasion by IL-1 was also confirmed in this study using cell culture on matrix-coated slip and 3D organotypic culture. Particularly, the infiltration length into collagen matrix by multicellular invasive cell strands was deepened by IL-1 treatment while the invasion length was diminished by IL-1R antagonist. Fascin depletion also inhibited cancer invasion in both experiments.

Increased expression of fascin, by TGF- $\beta$, has been also reported in gastric cancer as a Smad3 phosphorylationdependent response (21). Fascin expression by TGF $\beta$-Smad4 signal is abrogated through inhibition of DNA binding of Smad4 by transcription factor GATA3 in breast cancer cells (22). In addition, induction of fascin expression by IL-6 and TNF- in metastatic breast cancer cells has been reported (23). However, the cellular mechanism of fascin expression induced by $\mathrm{IL}-1 \beta$ has not been reported yet. In this study, mRNA expression of fascin was inhibited by biological-specific inhibitors PD98059, SP600125, parthenolide, and CREB inhibitor. In western blot analysis, IL-1 induced phosphorylation of ERK1/2, JNK, NF-KB and CREB. Their activation was markedly reduced by the IL-1R antagonist. These results indicate that ERK1/2, JNK, NF-KB, and CREB are cloasely invoved in fascin expression. Al-Tweigeri et al. reported that fascin is a key regulator of breast cancer invasion and NF-KB enhances its activity (24). Fascin knock-down cells have low NF-KB luciferase reporter activity after TNF-stimulation, whereas fascin-overexpressing cells showed significantly 
increase of NF-KB luciferase activity after TNF-stimulation. Snyder et al. have also observed that NF-KB requires STAT3 recruitment to the fascin promoter (23). Taken together, cAMP response element-binding protein (CREB) and aryl hydrocarbon receptor $(\mathrm{AhR})$ are associated with $\mathrm{FSCN} 1$ promoter region $(-219 /+114)$. They are also involved in the regulation of fascin transcription (25). These results indicate that fascin expression can be controlled through multiple stimuli and intracellular signal pathways during the invasion process and that NF-KB and CREB are crucial transcription factors in fascin expression.

In conclusion, in this study we observed that fascin expression was increased by IL- $1 \beta$ stimulation, leading to increased cancer invasion in oral squamous cell carcinoma. The regulation of cancer cells by the cancer microenvironment is a pivotal factor in cancer invasion and progression. Targeting the cancer microenvironment is improtant for cancer control.

\section{Conflicts of Interests}

There is no conflict of interests.

\section{Acknowledgements}

This research was supported by Basic Science Research Program through the National Research Foundation of Korea (NRF) funded by the Ministry of Education, Science and Technology (2015R1D1A1A01056946 and 2018R1D1A1B07042035).

\section{References}

1 Brabletz T, Kalluri R, Nieto MA and Weinberg RA: EMT in cancer. Nat Rev Cancer 18: 128-134, 2018.

2 Eddy RJ, Weidmann MD, Sharma VP and Condeelis JS: Tumor Cell Invadopodia: Invasive Protrusions that Orchestrate Metastasis. Trends Cell Biol 27: 595-607, 2017.

3 Lane J, Martin T, Weeks HP and Jiang WG: Structure and role of WASP and WAVE in Rho GTPase signalling in cancer. Cancer Genomics Proteomics 11: 155-165, 2014.

4 Weidle UH, Birzele F, Kollmorgen $G$ and Rueger R: Mechanisms and Targets Involved in Dissemination of Ovarian Cancer. Cancer Genomics Proteomics 13: 407-423, 2016.

5 Gould CM and Courtneidge SA: Regulation of invadopodia by the tumor microenvironment. Cell Adh Migr 8: 226-235, 2014.

6 Lucien F, Brochu-Gaudreau K, Arsenault D, Harper K and Dubois CM: Hypoxia-induced invadopodia formation involves activation of NHE-1 by the p90 ribosomal S6 kinase (p90RSK). PLoS One 6: e28851, 2011.

7 Rosenthal C Karlsson: pH-dependent invadopodia control. Nature Cell Biology 14: 50, 2012.

8 van Horssen R, Buccione R, Willemse M, Cingir S, Wieringa B and Attanasio F: Cancer cell metabolism regulates extracellular matrix degradation by invadopodia. Eur J Cell Biol 92: 113-121, 2013.

9 Cao H, Eppinga RD, Razidlo GL, Krueger EW, Chen J, Qiang $\mathrm{L}$ and McNiven MA: Stromal fibroblasts facilitate cancer cell invasion by a novel invadopodia-independent matrix degradation process. Oncogene 35: 1099-1110, 2016.

10 Murphy DA and Courtneidge SA: The 'ins' and 'outs' of podosomes and invadopodia: characteristics, formation and function. Nat Rev Mol Cell Biol 12: 413-426, 2011.
11 Li A, Dawson JC, Forero-Vargas M, Spence HJ, Yu X, König I, Anderson K and Machesky LM: The actin-bundling protein fascin stabilizes actin in invadopodia and potentiates protrusive invasion. Curr Biol 20: 339-345, 2010.

12 Bowden ET, Coopman PJ and Mueller SC: Invadopodia: unique methods for measurement of extracellular matrix degradation in vitro. Methods Cell Biol 63: 613-627, 2001.

13 Magdalena M. Auret S, Dahler AL, Smith L, Wong CF, Popa C, Barnes LM, Coman W and Saunders NA: Alterations in gene expression associated with head and neck squamous cell carcinoma development. Cancer Genomics Proteomics 1: 137148, 2004.

14 Hwang YS, Park KK and Chung WY: Epigallocatechin-3 gallate inhibits cancer invasion by repressing functional invadopodia formation in oral squamous cell carcinoma. Eur J Pharmacol 715: 286-295, 2013.

15 Bremnes RM, Dønnem T, Al-Saad S, Al-Shibli K, Andersen S, Sirera R, Camps C, Marinez I and Busund LT: The role of tumor stroma in cancer progression and prognosis: emphasis on carcinoma-associated fibroblasts and non-small cell lung cancer. J Thorac Oncol 6: 209-217, 2011.

16 Shiga K, Hara M, Nagasaki T, Sato T, Takahashi H and Takeyama $\mathrm{H}$ : Cancer-associated fibroblasts: their characteristics and their roles in tumor growth. Cancers (Basel) 7: 2443-2458, 2015.

17 De Wever O and Mareel M: Role of tissue stroma in cancer cell invasion. J Pathol 200: 429-447, 2003.

18 Afonina IS, Müller C, Martin SJ and Beyaert R: Proteolytic processing of interleukin-1 family cytokines: variations on a common theme. Immunity 42: 991-1004, 2015.

19 Lewis AM, Varghese S, Xu H and Alexander HR: Interleukin-1 and cancer progression: the emerging role of interleukin-1 receptor antagonist as a novel therapeutic agent in cancer treatment. J Transl Med 4: 48, 2006.

20 Idris A, Ghazali NB and Koh D: Interleukin 1 $\beta$-A Potential Salivary Biomarker for Cancer Progression? Biomark Cancer 7: 25-29, 2015.

21 Li L, Cao F, Liu B, Luo X, Ma X and Hu Z: TGF- $\beta$ induces fascin expression in gastric cancer via phosphorylation of $\operatorname{smad} 3$ linker area. Am J Cancer Res 5: 1890-1896, 2015.

22 Sun J, He H, Pillai S, Xiong Y, Challa S, Xu L, Chellappan S and Yang S: GATA3 transcription factor abrogates Smad4 transcription factor-mediated fascin overexpression, invadopodium formation, and breast cancer cell invasion. J Biol Chem 288: 36971-36982, 2013.

23 Snyder M, Huang J, Huang XY and Zhang JJ: A signal transducer and activator of transcription $3 \bullet$ Nuclear Factor $\mathrm{kB}$ (Stat $3 \bullet \mathrm{NFkB}$ ) complex is necessary for the expression of fascin in metastatic breast cancer cells in response to interleukin (IL)6 and tumor necrosis factor (TNF)- $\alpha$. J Biol Chem 289: 3008230089, 2014.

24 Al-Tweigeri T, Ajarim D and Al-Alwan M: Fascin is involved in the chemotherapeutic resistance of breast cancer cells predominantly via the PI3K/Akt pathway. Br J Cancer 111: 1552-1561, 2014.

25 Hashimoto Y, Loftis DW and Adams JC: Fascin-1 promoter activity is regulated by CREB and the aryl hydrocarbon receptor in human carcinoma cells. PLoS One 4: e5130, 2009.

Received September 7, 2018

Revised September 21, 2018

Accepted September 28, 2018 